\title{
INFLUENCE OF CARDIOPULMONARY BYPASS (CPB) ON SECRETION OF SEX STEROIDS
} IN CHILDREN

\author{
J. Enders ${ }^{1}$, H. Steckel ${ }^{1}$, C. Neuhäuser ${ }^{2}$, J. Thul ${ }^{3}$, D. Schranz ${ }^{3}$, M. Heckmann ${ }^{1}$ \\ ${ }^{1}$ General Pediatrics and Neonatology, University of Giessen, Giessen, Germany, ${ }^{2}$ Clinique Pediatrique, \\ Centre Hospitalier de Luxembourg, Luxemburg, Luxembourg, ${ }^{3}$ Pediatric Cardiology, University of Giessen, \\ Giessen, Germany
}

Background: Cardiac surgery with cardiopulmonary Bypass (CPB) leads to increased adrenal stress response. The role of androgens like testosterone or dihydrotestosterone (DHT) in mortality of critically ill adult patients is well known. In estrogens, antiinflammatory effects via inhibition of inflammatory cytokine transcription have been described and are associated with better neurological outcome. Data on postoperative metabolism of sex steroids after cardiac surgery on $\mathrm{CPB}$ are rare.

Aims: Does cardiac surgery with CPB lead to different sex steroids patterns in pediatric patients compared to surgery without CPB?

Methods: Prospective measurement of sex steroids (testosterone, DHT, estriol, 17-beta-estradiol, 17-OHpregnanolone, pregnanedial, pregnanetriol, 11-oxo-etiocholanolone ) in $24 \mathrm{~h}$ urine samples before and after surgery with CPB ( $\mathrm{n}=29$; median age 7.6 months; 8 girls; 5 ASD, 11 VSD, 6 TOF, 7 AVSD) compared to patient who underwent surgery without $\mathrm{CPB}$ ( $\mathrm{n}=19,8$ months; 3 girls).

Results: There were no significant differences in age, weight or gender. The CPB group showed a significant increase in DHT (Wilcoxon Test; Median 12.1 [Range 0-58.5] vs 6.6 [0-39.1]; $\mathrm{p}=0.0015$ ), estriol $(2.5[0-10.5]$ vs $1.3[0-11.8] \mathrm{p}=0.0023)$ and 17 beta-estradiol $(8.0[0-27.3]$ vs $0.5[0-8.6] ; \mathrm{p}<0.0001)$, and a trend towards higher testosterone $(4.60[0-27.82]$ vs $2.44[0-10.79] ; \mathrm{p}=0.0715)$.

Progesterone metabolites (17-OH-pregnanolone, pregnanetriol and 11-oxo-pregnanetriol) showed a stronger increase in the $\mathrm{CPB}$ group than in controls ( $\mathrm{p}=0.008 ; \mathrm{p}=0.022 ; \mathrm{p}=0.005$, respectively).

Conclusions: Cardiac surgery with CPB leads to higher secretion of androgens, estradiol and progesterone compared to surgery without $\mathrm{CPB}$. These findings suggest an important role of sex steroids in pediatric cardiac surgery already in early childhood. 